\title{
Research on the Enterprise Performance Management Information System Development and Robustness Optimization based on Data Regression Analysis and Mathematical Optimization Theory
}

\author{
Lei Liu, Hua Yang \\ Business School, Hunan University, Changsha, Hunan, P.R. China, 410082
}

\begin{abstract}
Performance management is core part of enterprise human resources management, for any organization or enterprise a good, the secret to its success lies in the effective performance management. Through the enterprise performance management, it can motivate employees, improve employee satisfaction, will help the staff's personal goals combined with enterprise strategy, realize the goal of the enterprise and the balance of personal development, promote enterprise realize sustainable development. In this paper, we conduct research on enterprise performance management information system development and the robustness optimization based on data regression analysis and mathematical optimization theory. We firstly review the modern regression analysis approaches for large-scale data with the introduction of our proposed revised regression model for enterprises. Later, we summarize the state-of-the-art mathematical optimization models to select the most feasible one to serve as the tool for our system. Then, we analyze the performance of the current existing performance management information system and adopt the prior knowledge to optimize the system. The experimental result proves the feasibility and effectiveness of our system. The performance evaluation accuracy is enhanced from $86.7 \%$ to $93.5 \%$ and the system robustness is enhanced as well.
\end{abstract}

Keywords: Management Information System; System Security; Enterprise Performance; Data Regression Analysis; Mathematical Optimization; Robustness Optimization.

\section{Introduction}

Information management system is to provide the information, support, business operation, management and decision function of the system, it holds a lot of useful information, how to make use of this information, query function is an important and indispensable part. However, traditional information management system such as query maintenance difficulties, especially to increase some information with a greater difference between existing information types, information system must be changed by original storage structure or new storage structure, this will increase maintenance workload of software code, so that the system cannot meet the needs of the enterprises and institutions in a timely manner. Management information system construction in order to better for the management of the full and the accurate information, information is recorded in the social life of all kinds of information symbols and basic data, from the point of the current social management, information is an important object of social management and components management plays an important role in the modern society. The construction of information management system is the inevitable demand of the information management which is one of the important social public management system development directions. The current information management system in the public affairs 
management has made full application and its function mainly for following several aspects. (1) Information management system with functions of plan. Information management system in the process of management of the information, can be in various management levels according to the known information of the detailed management plan, it make plans to meet actual requirements of management, improve management efficiency of management. (2) Information management system with functions of auxiliary decision-making. Due to mastering a large amount of basic data information management system, and can according to practical we need to make a comprehensive utilization data, including the data modeling, it is concluded that the optimal mathematical model, so as to provide the corresponding basis for correct decision-making. (3) Information management system with functions of data processing. Because the application of computer technology, information management system has a certain advantage in the data processing, mainly collected data can be calculated, transmission, storage, processing and the output, ensure the accuracy of the data to greatest extent. (4) Information management system has forecast function [1-2]. Information management system can not only analyze the known data and processing and also can use mathematical calculation with known information as the conditions, through mathematical modeling and calculation, analysis and forecast the future development trend. In the figure one, we show the sample structure of MIS system [3-4].

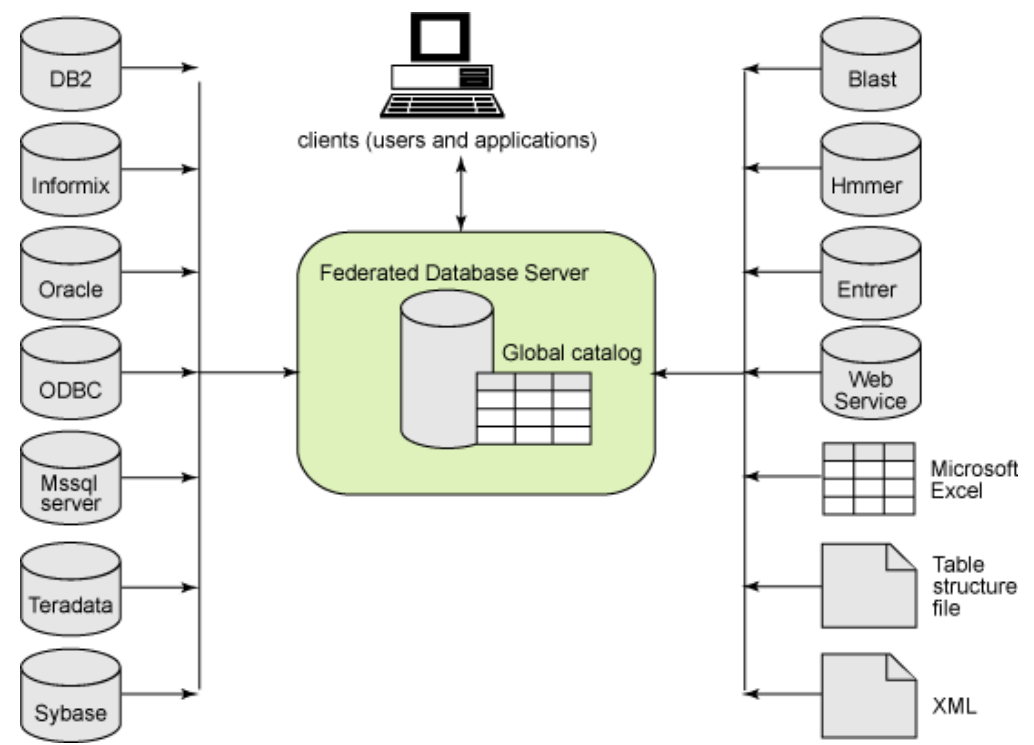

\section{Figure 1. The Sample Structure of the Management Information System Structure}

Information management system is to store huge amounts of data information, establish a good data storage structure to manage these large amounts of data, will affect the efficiency of information system and user satisfaction to the system. In traditional enterprise personnel management work, in the aspect of the development of people usually took the form of theory of endowment line. With the progress of the society, the establishment of modern enterprise human resources management, more and more companies tend to use based on performance of human resource development strategy. Subsequent, performance management has become the hot topic of human resource management [5-6].

Compensation allocation and performance evaluation is performance management, human resource planning and the basis of incentive mechanism and the foothold. In the traditional performance appraisal, different people were affected by same standards that are compared each other, but in modern performance, different people from different 
measure is compared with themselves. Qualitative analysis of knowledge sharing is given motivating individual knowledge sharing behavior guidelines as the implementation of incentive is needed based on quantitative assessment to ensure implementation of the enterprise knowledge management effect is of great significance, therefore which has become one of the problems of knowledge management research. Sufficient literature reviews has combined the mentioned aspects.

In [7], Tsai's group conducted research on the influence of the enterprise resource planning systems' performance on earnings management. For system evaluation is difficult to be the objective, comprehensive and accurate problem, puts forward the management information system of fuzzy comprehensive evaluation theory, the establishment of a system, a complete evaluation index system using the hierarchical clustering method to build up the management information system evaluation of class hierarchy using fuzzy hierarchical clustering method of the index weight, and set up a comprehensive evaluation is applicable to the management information system of multilevel fuzzy mathematics model. In [8], Sunil conducted research on the information management capability influences firm performance. Transaction driven method is studied in the application of management information system analysis of specific areas, construct a transaction tree, tree algorithm and discusses the construction affairs. The transaction tree is helpful to determine management information system in specific domain model element and the corresponding relation of element in the design space. In [9], Daniel conducted research on the supply chain integration and performance: the effects of long-term relationships, information technology and sharing, and logistics integration. To the operation of the data is an important content in management information system, using code automatic generation technology can effectively solve problem of code reuse, improve the development quality and efficiency of the management information system. More applications of the recent management information system could be found in [10-13].

In this paper, to enhance the performance and safety of tradition management information system for the performance evaluation, we conduct research on the enterprise performance management information system development and robustness optimization based on the data regression analysis and mathematical optimization theory. The reminding of the paper is organized as the follows. We firstly analyze the data regression methods to serve as the pre-analysis of the later discussion for the MIS system data optimization. Then, we introduce the mathematical optimization theory into the research and propose our new ones. Later, we briefly define the environment of developing MIS system and propose the detailed structure of our system with the optimization. Finally, we test the performance and conclude the work.

\section{The Regression Analysis for Large-scale Data}

\subsection{The Overview of the Regression Analysis}

Regression analysis and correlation analysis is the analysis of the two different methods. Correlation analysis has formed a set of theory with regression analysis, there is no complete theory can follow. Therefore the science and technology workers cross application in solving practical problems, to learn more and more complicated. Regression analysis is pop analytic geometry, the curve function. Scientific and technical workers in the practical work often get two series corresponding data with each of the other, draw a scatter diagram, intuitively in the middle of the scatterplot draw the smooth curve. Principal component regression analysis is a kind of in order to overcome the complex collinearity and using regression analysis method, in a linear regression model as the research object, uses the principal components estimate in parameter estimation, at the certain deviation at the expense of biased estimation [14-15]. 
In practice, especially in the process of chemical production, the mechanism of the object is not very clear, the dimensions of the input variable is very big, and there are serious of the collinearity, all sorts of direct solving method are encountered the insurmountable difficulties: due to the existence of collinearity could not exist. The lack of understanding of mechanism of object, nonlinear regression function form is very difficult to give and the increasing of the input nodes, the difficulty of neural network training also dramatically larger, and easy to cause a fitting problem. In addition, due to the large input dimension and the final regression model by means of direct robustness are relatively poor, this is because the models predict stability depends largely on model contains several independent variables.

In the formula one, we define the data compression regression analysis method. Input data compression basic idea is: when the output variables contribute information input variables, exists in the input data space of a linear or nonlinear subspace, can find a dimension less than the $\mathrm{p}$ subspace, and according to a certain point on the way of mapping the input data space corresponding to the projection space, can make the point of projection space in an acceptable way to explaining the corresponding output variable [16].

$$
\min \sum_{i=1}^{n}\left[a\left\|x_{i}-f\left(H\left(x_{i}\right)\right)\right\|^{2}+(1-a)\left\|y_{i}-\chi\left(H\left(x_{i}\right)\right)\right\|^{2}\right]
$$

We should define the parameters defined in the formula $2 \sim 4$ before conducting the further research. At first in order to guarantee the precision of the model, all may have an impact on the system output variables are listed in the input variables. Therefore it tends to have strong collinearity between the corresponding input variables.

$$
\begin{aligned}
& t=H(x), H: R^{p} \rightarrow R^{k} \\
& x=f(t), \quad f: R^{k} \rightarrow R^{p} \\
& y=\chi(t), \quad \chi: R^{k} \rightarrow R^{p}
\end{aligned}
$$

The principal component regression and the parameters in the main difference is that the general multivariate linear regression with the principal component regression using principal component estimation instead of least squares estimation. Principal component estimation is a biased estimation. In the Figure 2, we show the mentioned regression model.
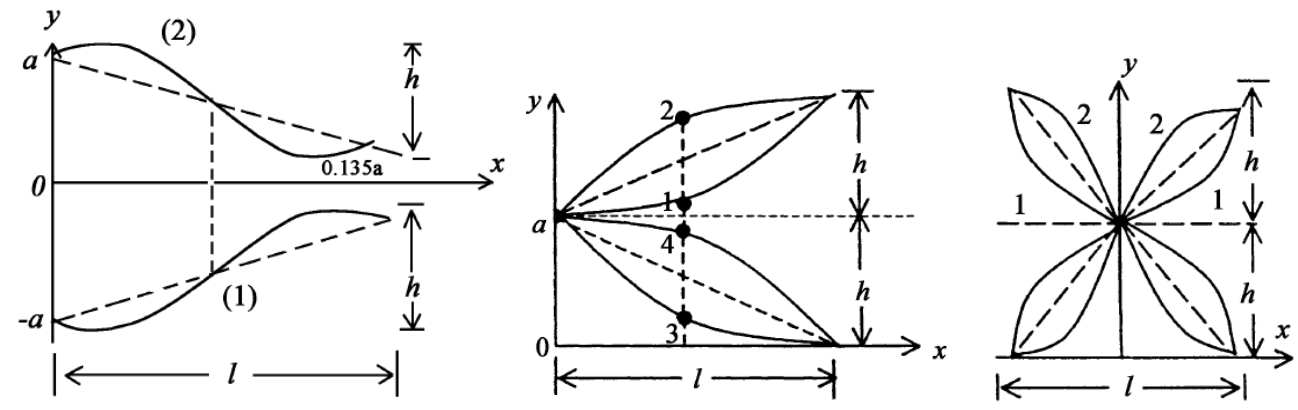

Figure 2. The Demonstration of the Regression Patterns

The basic method of principal component regression is by constructing linear combination of the original variables to produce a series of unrelated new variables, choose a few of new variables and make them as much as possible the original variable information, thus making use of this a few new variables instead of the original variable it possible to analyze and solve the problems. In the formula 5, we define the multiple linear regression models [17]. 


$$
Y_{\text {measurement }}=X_{\text {testing }} \beta+e, \quad e \sim N\left(0, \sigma^{2} I_{n}\right)
$$

In practice, sometimes to the centralized and standardized data as centralized data to make the sum of each column element of design matrix $X$ is zero. After standardization, we can use regression independent variables of relationship between $R$ analysis, eliminating the variable and the unit and value range, easy to estimate of the estimates of the regression coefficient of the analysis. After standardization center, the linear regression model is shown as the follows.

$Y^{\text {Centered }}=\beta_{0}+X^{\text {Centered }} \beta^{\text {Centered }}+e$

At this time, when using the principal component as new regression independent variables, the approximate principal component with zero impact on dependent variable can be ignored, so they can be removed from the regression model. Then using least square method to do the rest of principal component regression, and back to the original argument and got principal component regression. The figure three shows regression curves with difference algorithms.

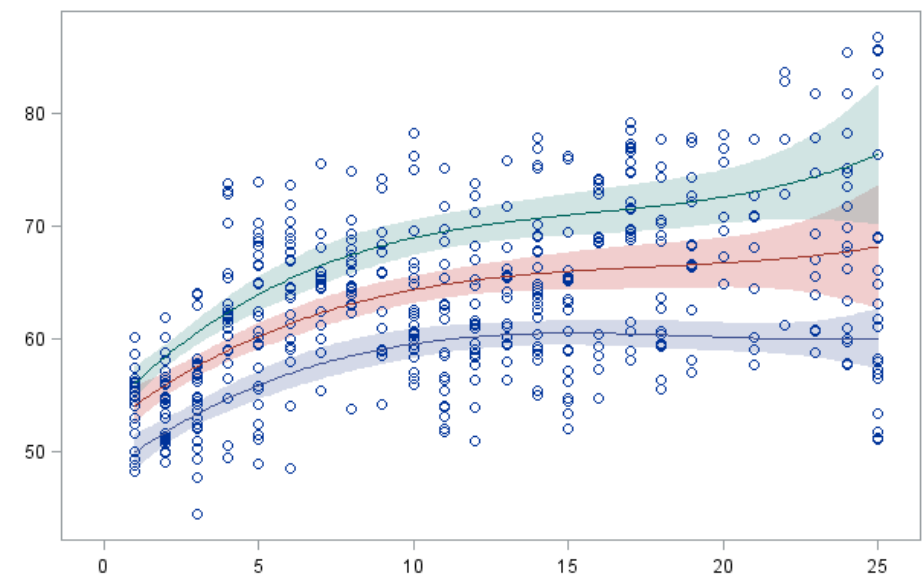

Figure 3. The Regression Curves with Difference Algorithms

\subsection{The Large-scale Data Regression Modification Approaches}

If the dependent variable is a composition data and associated with number of independent variables are also composition data, how to establish the multiple regression model between them is crucial. Typically, each data representation is a theme of meaning, and composition data composed of multiple components. In order to establish the regression model, a simple method is to list all of the component composition data together forming a large variable set, and then uses the ordinary variable analysis method for the processing. Obviously, the defect directly is difficult to explain the meaning of the different component data meaning and the function in the model. Therefore, in this design model in addition to variables and constraints, and complete the related questions, will also need we consider the hierarchy of the variables in the model. The formula 7 defines the reversion [18-19].

$Y_{i}=\beta_{0}+\beta_{1} X_{1 i}+\ldots+\beta_{k} X_{k i}+\varepsilon_{i}$

So use it for regression modeling can reflect the characteristics of the components as the model of interpretability and stronger. It has completely correlation data, making the ordinary least squares regression method in this case the complete failure, and therefore need to adopt modeling partial least-squares regression method. We revise the formula 7 into 8 . 
$Y_{i}=\mathrm{B}_{1}+\mathrm{B}_{2} \ln X_{i}+u_{i}$

The sum of the squares of the residuals is smaller, the better fitting effect, illustrates the relationship between the two variables, the more closely. But not only the size of the residual sum of squares and relevant experimental raw data, and also the nature of the fitting function, so should according to certain standard to collect the experimental data and try to eliminate the factors caused by residual, more important is according to the rule of the right to choose the appropriate fitting function shown as the following formula 9.

$$
\begin{aligned}
& \text { slope }=m=\frac{n\left(\sum x y\right)-\left(\sum x\right)\left(\sum y\right)}{n\left(\sum x^{2}\right)-\left(\sum x\right)^{2}} \\
& \text { int ercept }=b=\frac{\sum y-m\left(\sum x\right)}{n}
\end{aligned}
$$

Tests, although consistent test conditions, but the repeated measurement results of a certain parameter will fluctuate within a certain range and this is because the test site has random disturbance, caused the data of the stochastic volatility. So must go through before fitting to the original data preprocessing. Here, according to law of measured data, a given confidence interval in advance, if the sampling data is beyond this range, "for the singular value" that the sampling data is give out. The following formulas show the standard for selecting proper data.

$$
\begin{aligned}
& \mathrm{V}\left[\varepsilon_{\mathrm{i}}\right]=\mathrm{E}\left[\varepsilon_{\mathrm{i}}^{2}\right]=\sigma^{2} \\
& \operatorname{Cov}\left[\varepsilon_{\mathrm{i}}, \varepsilon_{\mathrm{j}}\right]=\mathrm{E}\left[\varepsilon_{\mathrm{i}}, \varepsilon_{\mathrm{j}}\right]=0
\end{aligned}
$$

\section{The Mathematical Optimization Theory}

\subsection{The Review of the Optimization and the Applications}

For a long time, the mathematicians, operational research, and the engineers to seek for the global optimal point paid a hard work, and achieved fruitful results, these results are basically divided into two directions, namely, determine the type of optimization method and random search optimization method. So-called determine the type of optimization method, it is using analytic properties, through the strict mathematical process to construct a certain converge to global optimal solution of point to get global optimal solution and these analytical properties including monotonicity, continuity, smoothness and convexity and so on. Determine the type of method is one of the biggest advantages is that it has a very good the optimality conditions, analytical properties for good questions also has good convergence and convergence speed. And its difficulty lies in the nature of the demand is higher, and to know in advance that these properties, in order to "suit the remedy to the case", and in engineering and practical problems in the optimization problem are often unpredictable, mostly can't satisfy method to determine the type of the objective function and the constraint condition of analytical requirements, or simply do not know its analytical nature. As a result, people turn to random search method to find the global optimal solution of the problem [20-23].

Due to these properties mainly reflects the local nature of the problem, for seeking the local optimal solution of the problem become a focus in the study of the early stage of the optimization method, now get a lot of strong convergence efficient algorithms, such as the Newton's method, the conjugate gradient method, quadratic programming method and the trust region method step by step. The method first to get the local optimal solution of the problem, and then come up to the global optimal solution from two directions, one is direct judgment of local optimal solution is global optimal solution, here is the main use of global convexity, monotonicity and or special problems such as the basic quadratic 
programming implementation; From the local optimal solution, to seek better local optimal solution, this kind of method is currently become the frontier of research method to determine the type, such as the tunnel function method and the filled function method, and the separation of programming method and the monotone method step by step. To finalize the optimization steps, we will need to test the methods on the data interpretation plane, in the following figure four, we demonstrate the plane that will be adopted later [24].

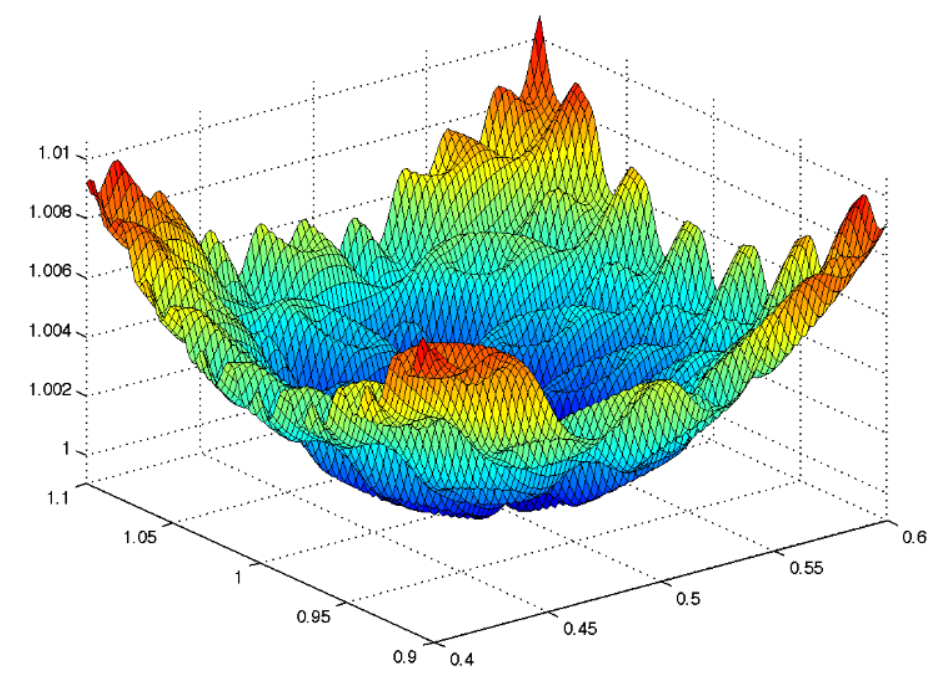

Figure 4. The Data Interpretation Plane for Optimizaation

\subsection{The Revised and Modified Optimization Algorithm}

Particle swarm optimization algorithm is a random optimization algorithm based on swarm intelligence, the basic idea is through the cooperation and competition between particles in a population of swarm intelligence to guide the optimization search and it has simple principle and the mechanism, to keep both the profound background of swarm intelligence evolution algorithm, and has a good optimization performance. The following formulas define the basic conditions of the algorithm [25].

$$
\begin{aligned}
& \text { lin } \Omega \equiv\left\{\sum \lambda_{j} \mathbf{a}_{j}: \mathbf{a}_{j} \in \Omega\right\} \\
& \text { aff } \Omega \equiv\left\{\sum \lambda_{j} \mathbf{a}_{j}: \mathbf{a}_{j} \in \Omega, \sum \lambda_{j}=1\right\} \\
& \text { cone } \Omega \equiv\left\{\sum \lambda_{j} \mathbf{a}_{j}: \mathbf{a}_{j} \in \Omega, \lambda_{j} \geq 0 \forall j\right\} \\
& \operatorname{conv} \Omega \equiv\left\{\sum \lambda_{j} \mathbf{a}_{j}: \mathbf{a}_{j} \in \Omega, \sum \lambda_{j}=1, \lambda_{j} \geq 0 \forall j\right\}
\end{aligned}
$$

Dynamic service composition technology is currently a hot research topic. In the process of the dynamic service composition need to choose the same function of multiple services and composite services to provide most suitable for the user's service and with the increasingly rich, provide services increase sharply to choose the scale of the problem. The traditional PSO algorithm in optimization of complex functions fashion to save a lot of shortcomings, such as poor local search ability and search accuracy is not high, easy to fall into the local optimum, search late shock, etc. Researchers from different angles on the traditional PSO algorithm are improved, in order to improve the algorithm performance. The following formulas define one.

$\left(c_{j}-\tilde{\mathbf{y}} \mathbf{A}_{j}\right) x_{j}=0 \quad \forall j=1, \ldots, n$ 
$\tilde{y}_{i}\left(\mathbf{A}^{i} \tilde{\mathbf{x}}-b_{i}\right)=0 \quad \forall i=1, \ldots, m$

An improved ant colony algorithm to optimize service portfolio and has good performance, but also can adapt to the dynamic nature of the combinatorial optimization problem. Here to choose the combination of ant colony algorithm to solve the above optimization problem. Through cooperation and competition between particles in a population of swarm intelligence to guide the optimization search, fast convergence speed. But in the early algorithms such as low accuracy, prone to defects under the condition of convergence, as a result of all particles to the optimal direction, the particle diversity, late significantly slow convergence speed and is easy to fall into local optimum. We optimize the traditional ones following the guideline.

$f(\hat{\mathbf{x}})=\max \{f(\mathbf{x}): \mathbf{x} \in \Omega\}$
$\hat{\mathbf{x}}=\operatorname{argmax}\{f(\mathbf{x}): \mathbf{x} \in \Omega\}$

The scope of the parameter $\Omega$ in the formulas should follow the restriction [26].

$\Omega=\left\{\mathbf{x} \in \mathrm{R}^{n}: \mathbf{g}^{i} \mathbf{x} \leq k_{i}(\right.$ for $i=1, \ldots, r), \mathbf{h}^{j} \mathbf{x}=l_{j}($ for $\left.j=1, \ldots, q)\right\}$

Chaos is a state of the rule-less movement, in a deterministic nonlinear system, without any additional random factor can generate the random behavior. Chaotic system has the special movement rule and main show is randomness, regularity and ergodicity. In the Figure 5, we show the final pattern of the mathematical optimization result.

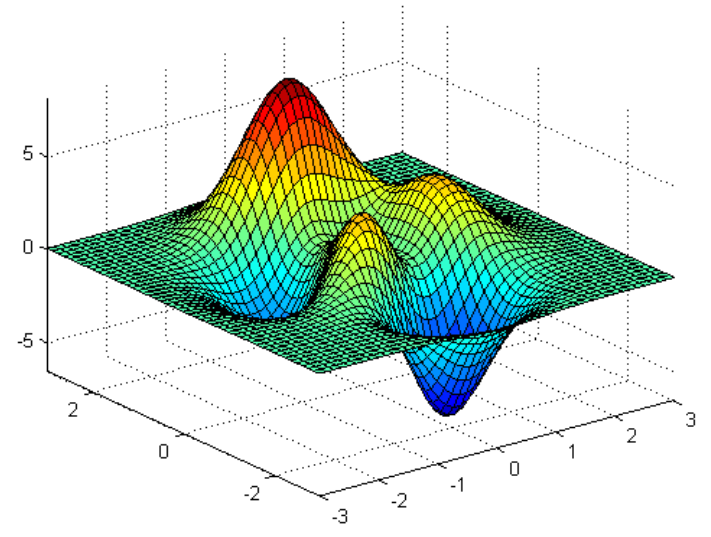

Figure 5. The Final Patternof the Mathematical Optimization Result

\section{The Enterprise Performance Management Information System}

\subsection{The Performance Evaluation Model}

Based on the value assessment is process integrity, emphasis on key value driving factors of enterprise, throughout the organization to improve the strategy and the business decisions. Based on the value assessment of the special attention to how to apply these concepts to make decision strategy and day-to-day operations, management implementation based on the value assessment of enterprise overall goal, analysis can be integrated with technology and the core management procedures promote enterprise management and decision making will focused on the value driving factors, to achieve its maximum value. The modern enterprise how to establish conforms to actual business operation performance evaluation system shown below. 
- Key performance evaluation indicators and targets set. Key performance indicators and targets set core and is the starting point of the performance evaluation cycle. Key performance indicators is set according to the enterprise the strategic target of the quantifiable objectives, once enterprise's strategic goal to determine key performance indicators can provide enterprises with a clear and intuitive method to measure the business enterprise the strategic goals to achieve or the not. In addition, the enterprise also needs to set for each key performance evaluation index of short-term and longterm goal, to define the success of enterprise standards and expectations for the future of enterprises, key performance indicators and goals for take a top-down approach.

- The establishment of enterprise strategy. Enterprise strategy is basis of performance evaluation cycle. Enterprise strategy for enterprise development provides a clear goal, is the other links in the performance evaluation cycle in order to achieve enterprise strategic objectives. Enterprise strategic objectives and key success factors for the enterprise's key performance evaluation index and target formulation provides the basis and direction [27-28].

- Monitoring and evaluation. Monitoring and the evaluation are based on performance targets for each department and process the actual performance of measurement and evaluation, timely understanding of enterprise internal operation and find the existing problems and gap. In setting performance goals at the same time, therefore, enterprise needs to determine the key performance evaluation indexes of specific weight, as a standard of performance evaluation for each assessment unit.

- Reward and guidance. Reward and guidance is last link of performance evaluation cycle. Incentives to link set up and running, performance evaluation cycle encourages enterprises within the correct behavior and motivate employees to achieve business goals and efforts, At the same time, by guiding the link set up and running, the performance evaluation cycle was carried out on problems arising from the enterprise internal operation and correct guidance in order to achieve the progress of enterprise.

Company management according to the development of the enterprise strategic target and combined with the company's mission and values, determine the enterprise level, function level and process level of key performance indicators and goals.

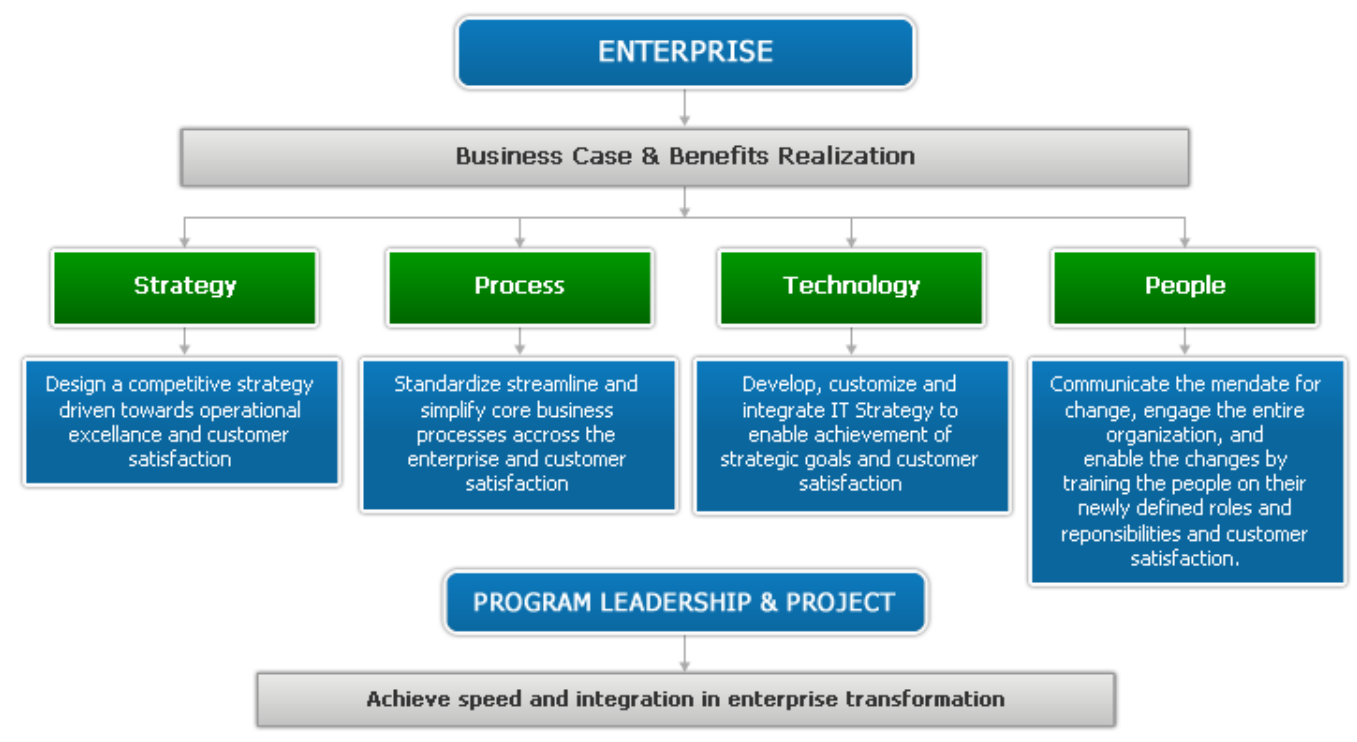

Figure 6. The Performance Evaluation Model and the Parameters 


\subsection{The Performance Model and the Management System Development}

Enterprise middle managers performance evaluation is a dynamic process, different due to different evaluation purpose or the same enterprise in different periods, different emphasis of the middle managers performance evaluation, which can lead to the determine differences in evaluation index weight and this is directly related to the accuracy of the assessment and the scientific features. There are many indicators in the performance evaluation index is as the descriptive, past the inspection, the indicators for the employee just a supporting role, is not really involved in the employee's performance appraisal, this is the disadvantages of previous performance evaluation. So we need to appraisal index and appraisal results are quantitative processing which is the purpose of the quantitative evaluation model is set up to quantify the performance appraisal results. The proposed algorithm is demonstrated in the Figure 7 as the basis of the prior discussion in the previous sections.

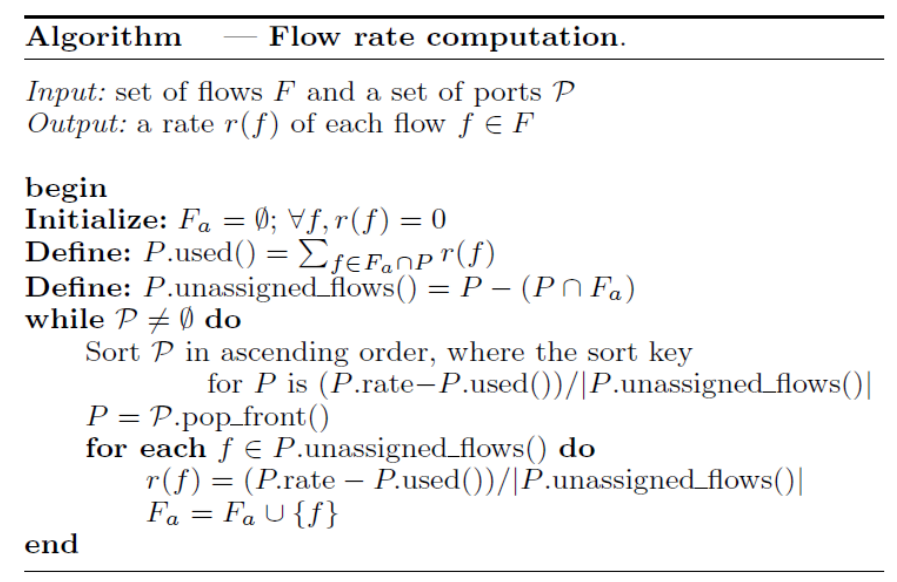

Figure 7. The Flowchart of the Proposed Algorithm

\section{Experiment}

In this part, we simulate the proposed method. The Figure 8 shows performance evaluation chain for further analysis. In this Figure, we mark all the necessary and potential elements for analysis with the discussion of the weight.

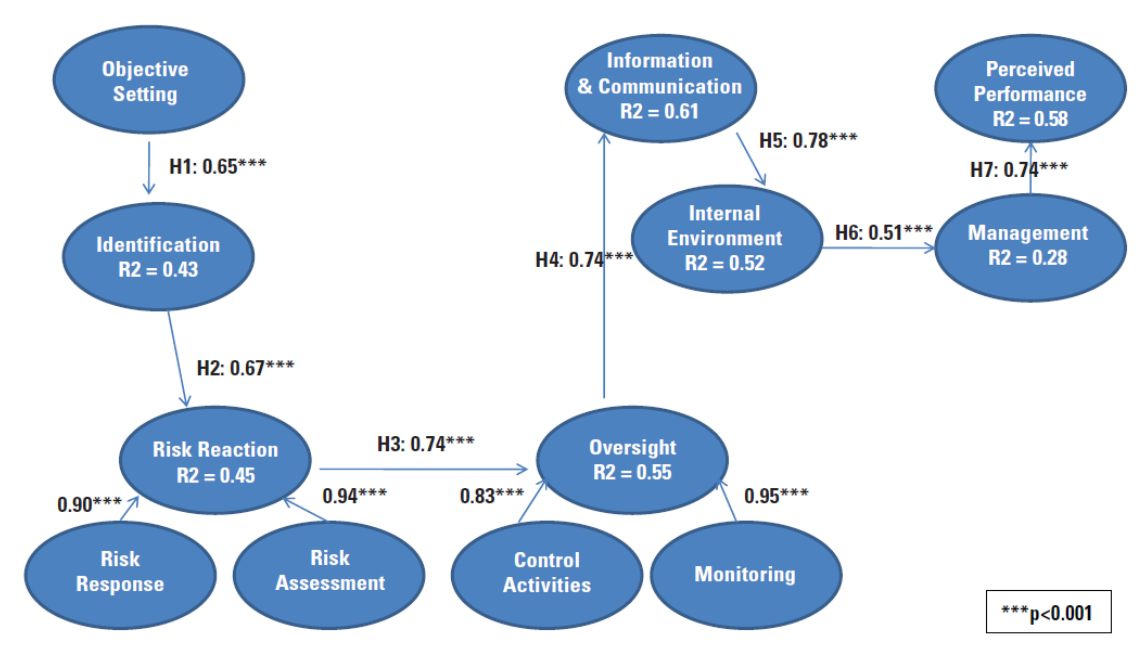

Figure 8. The Performance Evaluation Chain for Analysis 


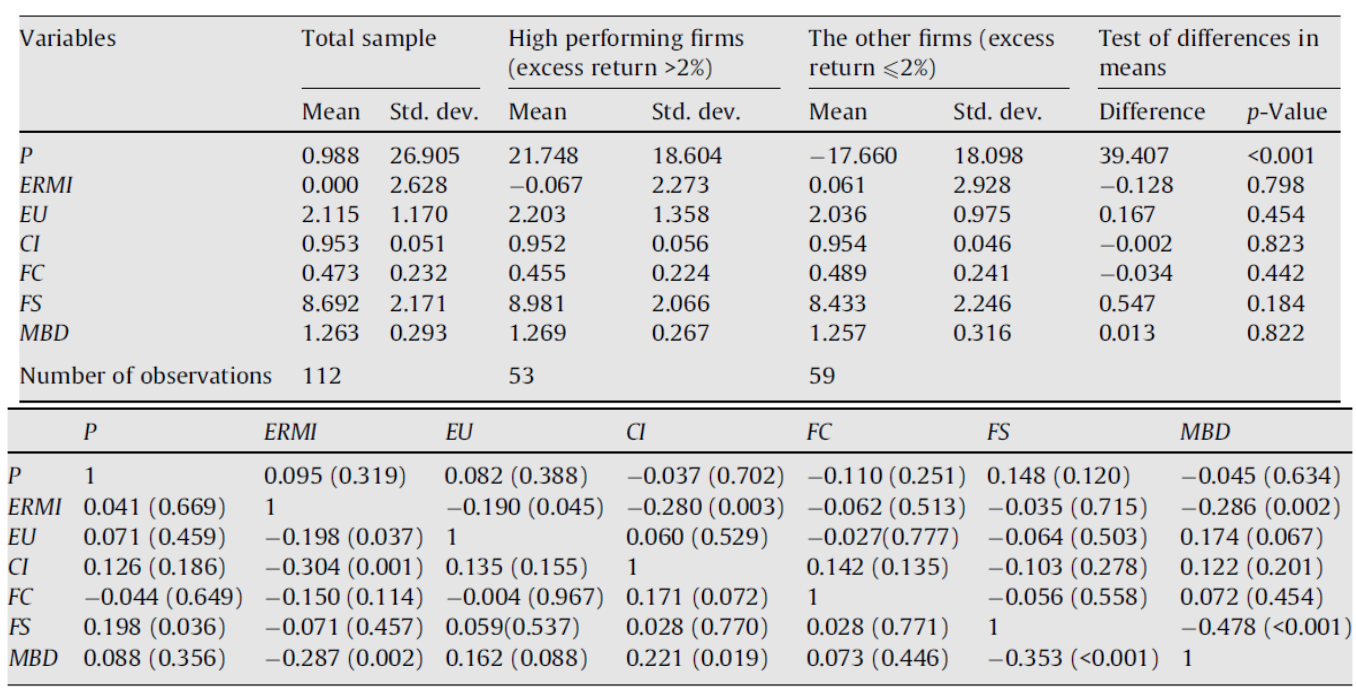

Figure 9. The Parameter Testing for the Proposed Methodology

\begin{tabular}{|c|c|c|c|c|c|c|}
\hline \multirow[t]{2}{*}{ Variables } & \multicolumn{2}{|c|}{ ERM firms } & \multicolumn{2}{|c|}{$\begin{array}{l}\text { Non-ERM matched } \\
\text { sample }\end{array}$} & \multicolumn{2}{|c|}{$\begin{array}{l}\text { Test of differences in } \\
\text { means }\end{array}$} \\
\hline & Mean & Std. dev. & Mean & Std. dev. & Difference & $p$-Value \\
\hline \multicolumn{7}{|c|}{ Panel A. Univariate test of differences in means } \\
\hline$P$ & 0.988 & 26.905 & 6.424 & 38.217 & -5.436 & 0.220 \\
\hline ERMI & 0.257 & 2.667 & -0.257 & 2.613 & 0.514 & 0.146 \\
\hline$E U$ & 2.115 & 1.170 & 1.997 & 1.186 & 0.118 & 0.455 \\
\hline $\mathrm{CI}$ & 0.953 & 0.051 & 0.948 & 0.089 & 0.006 & 0.564 \\
\hline$F C$ & 0.473 & 0.232 & 0.398 & 0.728 & 0.075 & 0.300 \\
\hline$F S$ & 8.692 & 2.171 & 8.311 & 2.502 & 0.382 & 0.224 \\
\hline$M B D$ & 1.263 & 0.293 & 1.147 & 3.995 & 0.115 & 0.761 \\
\hline Number of observations & 112 & 112 & & & & \\
\hline Number of observations & \multicolumn{2}{|c|}{ Total sample } & \multirow{2}{*}{\multicolumn{2}{|c|}{$\begin{array}{l}\text { High performing firms } \\
\text { (excess return }>2 \% \text { ) } \\
110\end{array}$}} & \multicolumn{2}{|c|}{$\begin{array}{l}\text { The other firms } \\
\text { (excess return } \leqslant 2 \% \text { ) }\end{array}$} \\
\hline & \multicolumn{2}{|c|}{224} & & & \multicolumn{2}{|c|}{114} \\
\hline Variables & \multicolumn{2}{|c|}{$\begin{array}{l}\text { Coefficients } \\
\text { ( } p \text {-value) }\end{array}$} & \multicolumn{2}{|c|}{ Coefficients ( $p$-value) } & \multicolumn{2}{|c|}{ Coefficients ( $p$-value) } \\
\hline
\end{tabular}

\section{Figure 10. The Performance Testing Across Different Systems and Approaches}

As demonstrated in the Figure 9, we illustrate parameter testing for proposed methodology. No matter how to divide the performance appraisal index, the assessment work must be done: assessment of indicators should be in as far as possible to quantifiable, the observed indexes is given priority to, should be more for behavioral description and not a description of the valuation. Evaluation indicators should as far as possible concise, without much too numerous in number, otherwise difficult to decide the size of the weight of each evaluation index. When determining the content of the assessment index, want to consider the actual characteristics and development of enterprise strategy, establish targeted and enterprise strategic corresponds to the index system to support enterprise strategic target realization. In the Figure 10, we show the performance testing across different systems and approaches. The result shows that our method outperforms the other approaches.

\section{Conclusion and Summary}

This research analyzes the enterprise performance management information system (MIS) development and robustness optimization based on data regression analysis and mathematical optimization theory. The development of information science and technology will promote the development of science and technology, economy and society, and the progress of science and technology, economy and the information 
technology changes have created the favorable conditions. In recent years, people are introducing new technology into information field, make information management of whole society, information retrieval, information analysis, reached a new level, promote the human civilization and economic development, vigorously promote the progress of society. Practice has proved that the computer information systems technology use can bring huge economic benefits and social benefits, but the development of an information system, must have a certain foundation, namely must have certain conditions and the conditions of reasonable application of these systems. Our research combines the core techniques of the data regression analysis and mathematical optimization theory to enhance the efficiency and performance robustness of the traditional systems. The experiment analysis proves the feasibility of our approach which will be meaningful.

\section{References}

[1] B, France, and R E. Crossler. "Privacy in the digital age: a review of information privacy research in information systems." MIS quarterly 35, no. 4 (2011), pp. 1017-1042.

[2] G Severin V., S A. Leech, and P J. Schmidt. "A review of ERP research: A future agenda for accounting information systems." Journal of information systems 25, no. 1 (2011):, pp. 37-78.

[3] L Seul-Ki, and J-H Yu. "Success model of project management information system in construction." Automation in construction 25 (2012), pp. 82-93.

[4] P Jagan A., and Sheryl R. Haut. "Patients with epilepsy and psychogenic non-epileptic seizures: an inpatient video-EEG monitoring study." Seizure 21, no. 1 (2012), pp. 24-27.

[5] A Amin. "Organizational Levels Model for Measuring the Effectiveness of Enterprise Resource Planning System (Case Study TUGA Company, Iran)." Universal Journal of Industrial and Business Management 2, no. 2 (2014), pp. 25-30.

[6] B, Edward WN, and Johann Mitlohner. "Characteristics of the multiple attribute decision making methodology in enterprise resource planning software decisions." Communications of the IIMA 5, no. 1 (2015), p.6.

[7] T, Wen-Hsien, K-C Lee, Jau-Yang Liu, Sin-Jin Lin, and Yu-Wei Chou. "The influence of enterprise resource planning (ERP) systems' performance on earnings management." Enterprise Information Systems 6, no. 4 (2012), pp. 491-517.

[8] M Sunil, N RAMASUBBU, and Va Sambamurthy. "How information management capability influences firm performance." MIS quarterly 35, no. 1 (2011), p. 237.

[9] P Daniel, and J Olhager. "Supply chain integration and performance: The effects of long-term relationships, information technology and sharing, and logistics integration." International Journal of Production Economics 135, no. 1 (2012), pp. 514-522.

[10] M, Konstantinos, and K Liagkouras. "Multiobjective evolutionary algorithms for portfolio management: A comprehensive literature review." Expert Systems with Applications 39, no. 14 (2012), pp. 1168511698.

[11] A Olubukola, P English, and J Pinkney. "Systematic review and meta-analysis of different dietary approaches to the management of type 2 diabetes." The American journal of clinical nutrition 97, no. 3 (2013), pp. 505-516.

[12] X, Li Da. "Information architecture for supply chain quality management." International Journal of Production Research 49, no. 1 (2011), pp. 183-198.

[13] Z, Abdel Nasser H. "An integrated success model for evaluating information system in public sectors." Journal of Emerging Trends in Computing and Information Sciences 3, no. 6 (2012), pp. 814-825.

[14] C Sierra, C. Ordóñez, A. Saavedra, and J. R. Gallego. "Element enrichment factor calculation using grain-size distribution and functional data regression." Chemosphere 119 (2015), pp. 1192-1199.

[15] Y Jinhong, and X Zhou. "Asymptotic theory in fixed effects panel data seemingly unrelated partially linear regression models." Econometric Theory 30, no. 02 (2014), pp. 407-435.

[16] L Eric Wai Ming. "An incremental adaptive neural network model for online noisy data regression and its application to compartment fire studies." Applied Soft Computing 11, no. 1 (2011), pp. 827-836.

[17] P, Nicholas G., and James G. Scott. "Data augmentation for non-Gaussian regression models using variance-mean mixtures." Biometrika (2013): ass081.

[18] S Tamer. "Data hiding in MPEG video files using multivariate regression and flexible macroblock ordering." Information Forensics and Security, IEEE Transactions on 7, no. 2 (2012, pp. 455-464.

[19] L Nengxiang, L Liang, and P Vieu. "Nonparametric regression estimation for functional stationary ergodic data with missing at random." Journal of Statistical Planning and Inference 162 (2015), pp. 75-87.

[20] Z Zhongshan, K Long, J Wang, and F Dressler. "On swarm intelligence inspired self-organized networking: its bionic mechanisms, designing principles and optimization approaches." Communications Surveys \& Tutorials, IEEE 16, no. 1 (2014), pp. 513-537. 
[21] S Herbert M., I R. Gil, M C. Graff, Jeff Wampler, German Merletti, Tie Sun, Hemali Patel et al. "3-D Hydraulic Fracturing and Reservoir Flow Modeling-Key to the Successful Implementation of a GeoEngineered Completion Optimization Program in the Eagle Ford Shale." Unconventional Resources Technology Conference (URTEC), (2015).

[22] B Nicolas, B Mishra, P-A. Absil, and Rodolphe Sepulchre. "Manopt, a Matlab toolbox for optimization on manifolds." The Journal of Machine Learning Research 15, no. 1 (2014), pp. 1455-1459.

[23] G Abhijit. "Simulation-Based Optimization: An Overview." In Simulation-Based Optimization, pp. 2935. Springer US, (2015).

[24] AlM. M., M Golalikhani, and J Zhuang. "A computational study on different penalty approaches for solving constrained global optimization problems with the electromagnetism-like method." Optimization 63, no. 3 (2014), pp. 403-419.

[25] N Angelia, and A Olshevsky. "Distributed optimization over time-varying directed graphs." Automatic Control, IEEE Transactions on 60, no. 3 (2015), pp. 601-615.

[26] G Kuidong, C Du, H Wang, and S Zhang. "An Efficient of Coal and Gangue Recognition Algorithm." International Journal of Signal Processing, Image Processing \& Pattern Recognition 6, no. 4,345 (2013): p. 354.

[27] L Huei, K Lane Chen, and J Yang. "Teaching enterprise resource planning (ERP) systems in the supply chain management course." Communications of the IIMA 6, no. 3 (2015), p. 8.

[28] Wang, Cheng-Hua, and Wen-Ya Tsai. "Elucidating How Interface Design and Cognitive Function Affect Learning Performance in the Enterprise Resource Planning (ERP) Software System." Journal of Testing and Evaluation 44, no. 1 (2014): 1-16. 
International Journal of Security and Its Applications

Vol. 10, No. 4 (2016)

s 